\title{
A core outcome set for clinical trials of chemoradiotherapy interventions for anal cancer (CORMAC): a patient and health-care professional consensus
}

\author{
Rebecca Fish, Caroline Sanders, Richard Adams, Julie Brewer, Sara T Brookes, Jill DeNardo, Rohit Kochhar, Mark P Saunders, \\ David Sebag-Montefiore, Paula R Williamson*, Andrew G Renehan*
}

Chemoradiotherapy is the primary treatment for patients with squamous cell carcinoma of the anus, but variations in the reported outcomes have restricted between-study comparisons. Treatment-related morbidity is considerable; however, no trial has comprehensively quantified long-term side-effects or quality of life. Therefore, we established the first international health-care professional and patient consensus to develop a core outcome set, using the Core Outcome Measures in Effectiveness Trials method. We used the results from our previous systematic review and combined them in this Review with patient interviews to derive a comprehensive list of outcomes, followed by a two-round Delphi survey completed by 149 participants (55 patients and 94 health-care professionals) from 11 countries. The Delphi results were discussed at a consensus meeting of health-care professionals and patients. Agreement was reached on 19 outcomes across four domains: disease activity, survival, toxicity, and life impact. Implementation of the Core Outcome Research Measures in Anal Cancer (CORMAC) set in future trials will serve as a framework to achieve standardisation, facilitate selection of health-area-specific evaluation tools, reduce redundancy of outcome lists, allow between-study comparisons, and ultimately enhance the relevance of trial findings to healthcare professionals, trialists, and patients.

\section{Introduction}

The incidence of squamous cell carcinoma of the anus has increased globally over the past three decades, ${ }^{1}$ most markedly in high-income countries, where the standardised incidence is $0.4-1.8$ per 100000 people. Historically, radical surgical resection was the primary treatment for squamous cell cancer of the anus, until a framework shift occurred in the 1970s and 1980s when small nonrandomised cohort studies showed high levels of local control with primary radiotherapy, with or without chemotherapy (fluorouracil with mitomycin C), ${ }^{2,3}$ and an opportunity for anal sphincter preservation. Subsequently, six phase 3 randomised trials (2877 patients) in Europe and the USA established the effectiveness of chemoradiotherapy as primary treatment. ${ }^{49}$ Over this period (1990s onwards), survival improved progressively; 5 -year overall survival approached $75 \% .^{10}$ However, this success has come at the cost of considerable treatmentrelated acute and long-term toxicity, because of the high radiation doses needed for treatment and unavoidable irradiation of adjacent structures in the pelvis.

Across the aforementioned six trials, variation in the outcomes reported is considerable, limiting betweenstudy comparisons and delaying the progress in evidence collection. ${ }^{11}$ Furthermore, outcomes in these trials were primarily related to survival and disease activity, and no trial comprehensively addressed long-term side-effects or quality of life (QoL). Both issues can be addressed by the development of a core outcome set, "an agreed, standardised collection of outcomes which should be measured and reported, as a minimum, in all trials for a specific clinical area". ${ }^{12}$ The core outcome set has been endorsed as a means to reduce outcome heterogeneity, and to increase the relevance of research through the involvement of key stakeholders in its development. ${ }^{13}$ This Review describes the development of a core outcome set for trials of chemoradiotherapy interventions for squamous cell carcinoma of the anus.

\section{Methods \\ Study overview}

The scope of the core outcome set was defined according to the criteria recommended by Core Outcome Measures in Effectiveness Trials (COMET) $:{ }^{13}$ the health condition was non-recurrent, non-metastatic squamous cell carcinoma of the anus; the population was adults aged older than 18 years; the type of intervention was primary treatment with chemoradiotherapy; and the setting was later phase (phase 2 or 3) trials that will inform clinical decision making. The core outcome set was developed in three phases, inclusive of patients and healthcare professionals at each stage: (1) a long list of outcomes was generated through systematic review ${ }^{14}$ and semistructured patient interviews; (2) the long outcome list was used to populate a two-phase Delphi process; and (3) the results of the Delphi survey were reviewed at a consensus meeting and a final core outcome set was determined.

\section{Gathering information}

Outcomes of importance to patients were identified through semi-structured interviews. This approach uses open questions to facilitate a patient-led discussion, guided by additional prompts from a pre-prepared topic guide to ensure key areas are covered. Patients for the semi-structured interviews were identified and recruited from the Christie National Health Service (NHS) Foundation Trust (Manchester, UK) anal cancer database
Lancet Gastroenterol Hepatol 2018; 3: 865-73

*Joint senior authors

Division of Cancer Sciences, School of Medical Sciences, Faculty of Biology, Medicine and Health (R Fish FRCS, Prof A G Renehan PhD), Centre for Primary Care

(C Sanders PhD), and Manchester Cancer Research Centre and National Institute for Health Research, Manchester Biomedical Research Centre (Prof A G Renehan), University of Manchester, Manchester, UK; Colorectal and Peritoneal Oncology Centre, Christie National Health Service Foundation Trust, Manchester UK (R Fish, ) Brewer HipHE, J DeNardo, R Kochhar FRCR, M P Saunders PhD, Prof A Renehan); Centre for Trials Research, Cardiff University School of Medicine, Cardiff, UK (Prof R Adams FRCR); Velindre Cancer Centre, Cardiff, UK (Prof R Adams); Cancer Research UK Clinical Trials Unit, Institute of Cancer and Genomic Sciences, University of Birmingham, Birmingham, UK (ST Brookes PhD); Leeds Cancer Centre, University of Leeds, St James University Hospital, Leeds, UK (Prof D Sebag-Montefiore FRCR); and Medical Research Council North West Hub for Trials Methodology Research, Department of Biostatistics, University of Liverpool, Liverpool, UK (Prof P R Williamson PhD) Correspondence to: Ms Rebecca Fish, Division of Cancer Sciences, School of Medical Sciences, Faculty of Biology, Medicine and Health, University of Manchester, Manchester M13 9GB, UK rebecca.fish@doctors.org.uk 
and through the Macmillan anal cancer forum, ${ }^{15}$ following a purposive sampling matrix defined a priori. Eligibility criteria and the sampling matrix are available in the study protocol. ${ }^{16}$ Written informed consent was obtained before interviews. Outcomes were identified both indirectly, through listening to patients' experiences, and directly, by asking about their treatment information needs before, during, and after treatment. Audio recordings of the interviews were transcribed in full and coded to identify outcomes.

Outcomes from the systematic review and patient interviews were combined to generate the long list of outcomes. This list, along with relevant quotes from patients' interviews, was discussed by the CORMAC study advisory group, in September, 2017. For each outcome, the group agreed to merge closely related items, to exclude outcomes considered to be of limited clinical importance (eg, extremely rare events) and not identified in the patient interviews, and to ensure face validity and domain allocation. The final outcome list was used to populate the Delphi questionnaire. Outcomes were converted into question items, with clinical and plain language versions, which were reviewed for face validity, understanding, and acceptability by the Christie NHS Foundation Trust Patient Information Committee, comprising health professionals and lay members, and modified according to feedback.

\section{Delphi survey}

We ran the Delphi survey with the online DelphiManager platform. ${ }^{17}$ Participants were recruited from the two key stakeholder groups: patients and health-care professionals. Clinical researchers involved in clinical trials formed a subgroup within the health-care professional stakeholder group. Patients were recruited from four UK treatment centres, social media (Twitter), and patient advocacy groups (appendix p 2). Patients were asked to declare that they had received, or were receiving, treatment with chemoradiotherapy for squamous cell carcinoma of the anus, when registering to take part in the Delphi process. Health-care professionals were recruited by e-mail to principal investigators in the PersonaLising Anal cancer radioTherapy dOse (PLATO) trial ${ }^{18}$ and via UK and international professional organisations (appendix p 2). Eligibility criteria are detailed in the study protocol. ${ }^{16}$ The Delphi process was done over two rounds. In each round, participants were asked to rate the importance of including each outcome in the core outcome set on a 1-9 scale described as: limited importance (1-3), important but not critical (4-6), and critically important (7-9). Participants could suggest additional outcomes at the end of round one, which were reviewed by the core team (RF, CS, AGR, and PRW). Any outcome not already represented was added to round two. No outcomes were removed between rounds. In round two, participants were shown a histogram of the round one scores for each outcome together with their own round one score, before being asked to reflect on the information presented and score each outcome again.

The percentage of participants scoring the importance of each outcome on a scale of 1-9 was calculated from the scores obtained during round two. Consensus criteria were defined a priori: ${ }^{12}$ outcomes scored as critically important (7-9) by 70\% or more of patients and $70 \%$ or more of health-care professionals, and limited importance $(1-3)$ by $15 \%$ or less of patients and $15 \%$ or less of health-care professionals, were defined as having reached consensus for inclusion (consensus in) and were included in the provisional core outcome set. Outcomes scored $1-3$ by $70 \%$ or more and $7-9$ by $15 \%$ or less of both stakeholder groups were defined as having reached consensus for exclusion (consensus out) and were excluded. Outcomes not fulfilling criteria for consensus in or out were defined as not having reached consensus between health-care professionals and patients (no consensus).

\section{Consensus meeting}

The results of the Delphi survey were presented at a consensus meeting. Participants were eligible to attend if they had completed both rounds of the Delphi survey. Participants were sampled to achieve a balanced representation of patients and health-care professionals of different disciplines. International participation was restricted because of budgetary constraints. Before the meeting, all participants were sent a summary of their own Delphi round two scores. The meeting was chaired by an independent, non-clinical researcher, with expertise in core outcome set development methodology (STB), and who was not a member of the study advisory group.

Outcomes identified in round two of the Delphi as having reached consensus for inclusion were presented first and participants were asked if there were any fundamental reasons why these should not be included in the core outcome set. Outcomes deemed to be having reached consensus for exclusion were reviewed and participants were asked if there were any fundamental reasons why these should be included in the core outcome set. All outcomes for which no consensus was reached were discussed and voted on. Outcomes that scored 7-9 by $70 \%$ or more of one stakeholder group were considered first. The remaining no consensus outcomes were reviewed together, with individual outcomes being discussed and voted on, only if proposed as being important by a meeting participant. Contrasting views were actively sought and the chair ensured all participants had equal opportunity to contribute before voting commenced. Voting was done anonymously by use of TurningPoint software and handsets (Turning Technologies LLC, Youngstown, Ohio, OH, USA). Voting and consensus criteria followed the same format as in the Delphi survey, with results displayed to participants immediately for each 
outcome. Outcomes meeting the criteria for consensus through voting were included in the core outcome set; all other items were excluded. At the end of the meeting, the final core outcome set was presented to participants and ratified.

\section{Other analyses}

We assessed for attrition bias between Delphi round one and round two, comparing the distribution of mean round one scores between participants who did and did not complete round two. ${ }^{12}$ We assessed for consensus meeting participation bias by comparing the distribution of mean round two scores between participants who did and did not participate in the consensus meeting. To assess satisfaction with the process and outcome of the consensus meeting, we collected a feedback questionnaire from participants (appendix pp 3-5).

\section{Ethics and registration}

Our findings are reported in line with the Core Outcome Set-Standards for Reporting (COS-STAR) guidance. ${ }^{19}$ This project was prospectively registered with the COMET initiative. ${ }^{20}$ The study protocol and composition of the study advisory group have been published elsewhere. ${ }^{16}$ The study was approved by the National Research Ethics Service (semi-structured interviews: IRAS ID 183034, CPMS study ID 20368, adopted in January, 2016; Delphi and consensus meeting: IRAS ID 215791, CPMS Study ID 33052, adopted in February, 2017).

\section{Results}

\section{Information gathering}

The systematic review has been described in detail elsewhere. ${ }^{14}$ Briefly, 1243 outcomes were identified from 101 studies, consolidated into 92 standardised outcome terms (figure). Interviews with 19 patients identified 61 outcomes, including eight not identified from the literature (skin pain, skin itch, sleep disturbance, bone or joint pain, fertility, menopause, ejaculatory function, and orgasmic function). The 100 standardised outcome terms were categorised into five domains (survival, disease activity, life impact, delivery of care, and toxicity), which can be mapped directly to the outcome domain taxonomy recommended by COMET. ${ }^{21}$ After discussion by the study advisory group, 73 standardised outcome terms were taken forward into the Delphi process; one outcome was expanded into two and 28 were removed (appendix pp 6-15).

\section{Delphi process}

149 participants from 11 countries (55 patients and 94 health-care professionals) completed both rounds of the Delphi process (table 1). 30 additional outcomes were proposed during round one, of which five were added into round two, and two outcome descriptions were revised (appendix pp 16-20). The full list of Delphi question items is available in appendix pp 21-26.

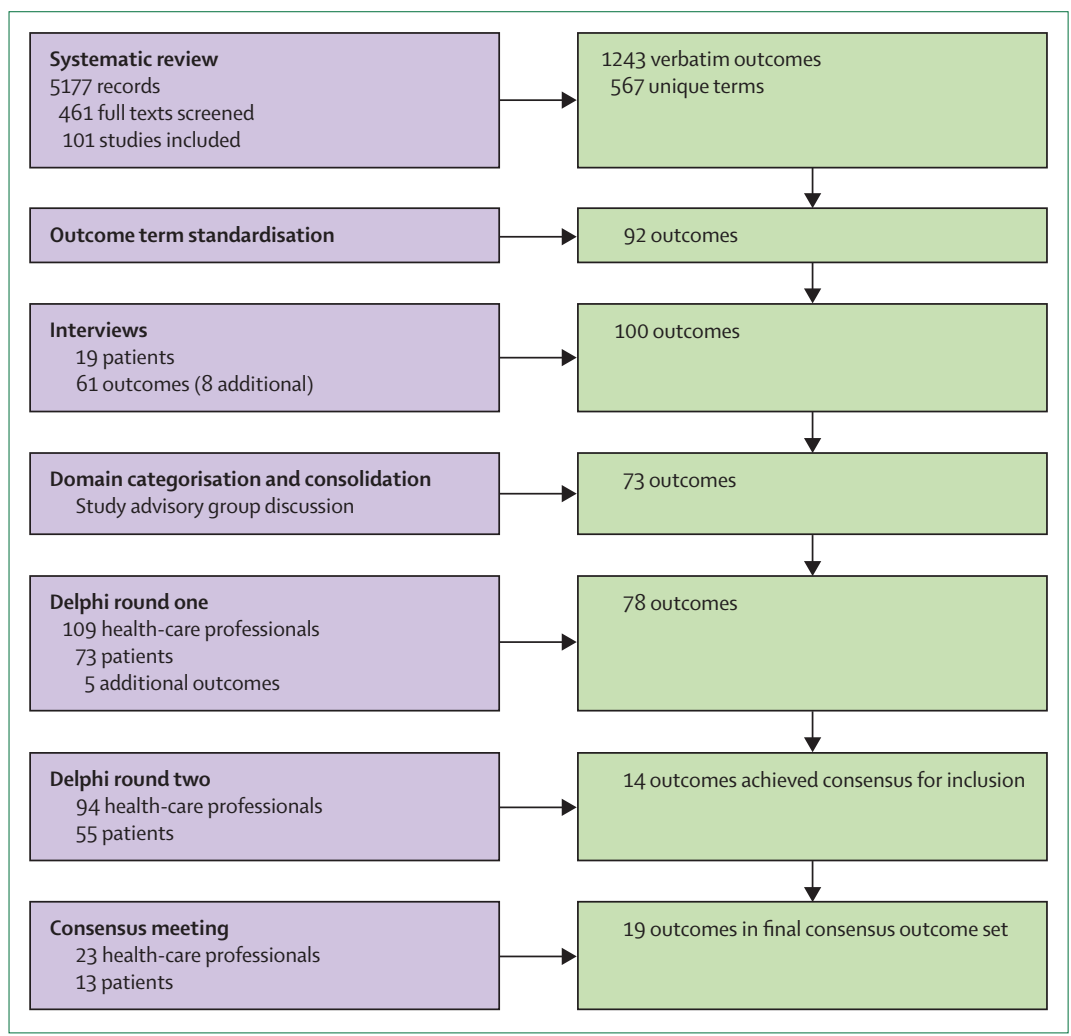

Figure: Overview of the core outcome set development

14 outcomes met the criteria for having reached consensus for inclusion - these were treatment response, local failure, regional failure, distant failure, disease progression, salvage surgery, disease-free survival, metastasis-free survival, cancer-specific survival, progression-free survival, health-related QoL (HRQoL), anal incontinence, colonoscopy/ileoscopy, and pelvic fistula. No outcomes met the original criteria for having reached consensus for exclusion so it was agreed by the study advisory group to redefine the criteria for having reached consensus for exclusion to a majority ruleie, outcomes were classed as having reached consensus for exclusion if $50 \%$ or less of participants in both stakeholder groups scored the item as critically important (7-9). 13 outcomes met the revised criteria for having reached consensus for exclusion (appendix 27-36). 51 outcomes did not meet consensus criteria.

The attrition rate from round one to round two was $18 \%$ (33 of 182 participants; $14 \%$ [15 of 109] of health-care professionals, $25 \%$ [18 of 73] of patients). Comparison of mean round one scores between participants who did (7 . 25 [SD 1.29] for patients and 6.46 [0.98] for healthcare professionals) and did not complete both rounds $(8 \cdot 18[0 \cdot 82]$ for patients and $7 \cdot 01$ [0.89] for health-care professionals) suggested that those who did not complete round two could have been more likely to score all outcomes higher than those continuing to round two (appendix p 1). Patients recruited via social 
media and group emails through patient advocacy groups (33 [60\%] of 55 patients) had a higher attrition rate than did those recruited via hospital sites (15 [31\%] of 48 vs four [15\%] of 26).

\section{Consensus meeting}

23 health-care professionals and 13 patients participated in the consensus meeting (appendix pp 37-38). Only six patients who completed both rounds of the Delphi accepted the invitation to the consensus meeting, therefore to help balance patient and health-care professionals numbers, the eligibility criteria for patient participants was expanded to include patients who had taken part in the round one interviews. Comparison of the mean round two scores of participants attending with those not attending the consensus meeting suggests no participation bias, although numbers involved in round two and the consensus meeting are low (appendix p 1).

During discussions, participants suggested that different aspects of sexual function can be important to

\begin{tabular}{|c|c|}
\hline & $\mathrm{n}(\%)$ \\
\hline Health-care professionals & $94(100 \%)$ \\
\hline \multicolumn{2}{|l|}{ Involvement in clinical trials } \\
\hline $\begin{array}{l}\text { Named author on a published } \\
\text { phase } 3 \text { randomised trial in anal cancer }\end{array}$ & $5(5 \%)$ \\
\hline $\begin{array}{l}\text { Working group for an ongoing or planned } \\
\text { randomised trial in anal cancer }\end{array}$ & $9(10 \%)$ \\
\hline Other anal cancer trial involvement & $45(48 \%)$ \\
\hline Not directly involved in anal cancer trials & $34(36 \%)$ \\
\hline Missing & $1(1 \%)$ \\
\hline \multicolumn{2}{|l|}{ Occupation } \\
\hline Coloproctologist & $36(38 \%)$ \\
\hline Oncologist & $26(28 \%)$ \\
\hline Infectious diseases clinician & $4(4 \%)$ \\
\hline Pathologist & $4(4 \%)$ \\
\hline Radiographer & $6(6 \%)$ \\
\hline Radiologist & $5(5 \%)$ \\
\hline Radio-physicist & $1(1 \%)$ \\
\hline Specialist nurse & $11(13 \%)$ \\
\hline Missing & $1(1 \%)$ \\
\hline \multicolumn{2}{|l|}{ Country of residence } \\
\hline Australia & $10(11 \%)$ \\
\hline Canada & $1(1 \%)$ \\
\hline France & $1(1 \%)$ \\
\hline Netherlands & $2(2 \%)$ \\
\hline New Zealand & $2(2 \%)$ \\
\hline Norway & $1(1 \%)$ \\
\hline Spain & $1(1 \%)$ \\
\hline Sweden & $1(1 \%)$ \\
\hline UK (England) & $58(62 \%)$ \\
\hline UK (Scotland) & $2(2 \%)$ \\
\hline UK (Wales) & $9(10 \%)$ \\
\hline \multirow[t]{2}{*}{ USA } & $6(6 \%)$ \\
\hline & ontinues in next column) \\
\hline
\end{tabular}

different people, hence no individual outcome would reach the threshold for inclusion, despite broad agreement that overall sexual function should be included. Participants proposed and agreed through voting that all outcomes related to sexual function should be grouped together, under a single broader outcome called sexual function. This term mirrors other functional outcomes considered (physical, emotional, role or occupational, and social function). This decision was subsequently validated on examination of the round two results, which showed that $44(80 \%)$ of 55 patients and 67 (71\%) of 94 health-care professionals scored 7-9, for at least one outcome in the sexual and reproductive toxicity domain (table 2).

The new sexual function outcome and four outcomes for which no consensus had been reached between healthcare professionals and patients in the Delphi survey-ie, overall survival, physical function, faecal urgency, and desquamation-reached the criteria for achieving consenus for inclusion after discussion and voting at the meeting and were included in the final core outcome set. Six outcomes that did not reach the threshold for consensus in were scored as critically important (7-9) by $70 \%$ or more of patients (cognitive function, emotional function, occupational or role function, anal pain, gastrointestinal [anorectal] bleeding, and vaginal toxicity).

\begin{tabular}{|lc|}
\hline & $\mathbf{n}(\%)$ \\
\hline (Continued from previous column) & $55(100 \%)$ \\
Patients & \\
Time since completion of treatment & $5(9 \%)$ \\
Still undergoing treatment & $8(15 \%)$ \\
Within the last 6 months & $31(56 \%)$ \\
Within the last 5years & $11(20 \%)$ \\
More than 5years ago & \\
Sex & $47(85 \%)$ \\
Female & $7(13 \%)$ \\
Male & $1(2 \%)$ \\
Missing & \\
Sexuality & $50(91 \%)$ \\
Heterosexual & $4(7 \%)$ \\
Homosexual & $1(2 \%)$ \\
Missing & \\
Age & $25(45 \%)$ \\
30-60 years & $33(60 \%)$ \\
>60 years & $22(40 \%)$ \\
Country of residence & \\
Australia & \\
Canada & \\
Netherlands & \\
UK (England) & \\
UK (Wales) & \\
USA & \\
\hline completed both rounds of the Delphi process & \\
\hline
\end{tabular}




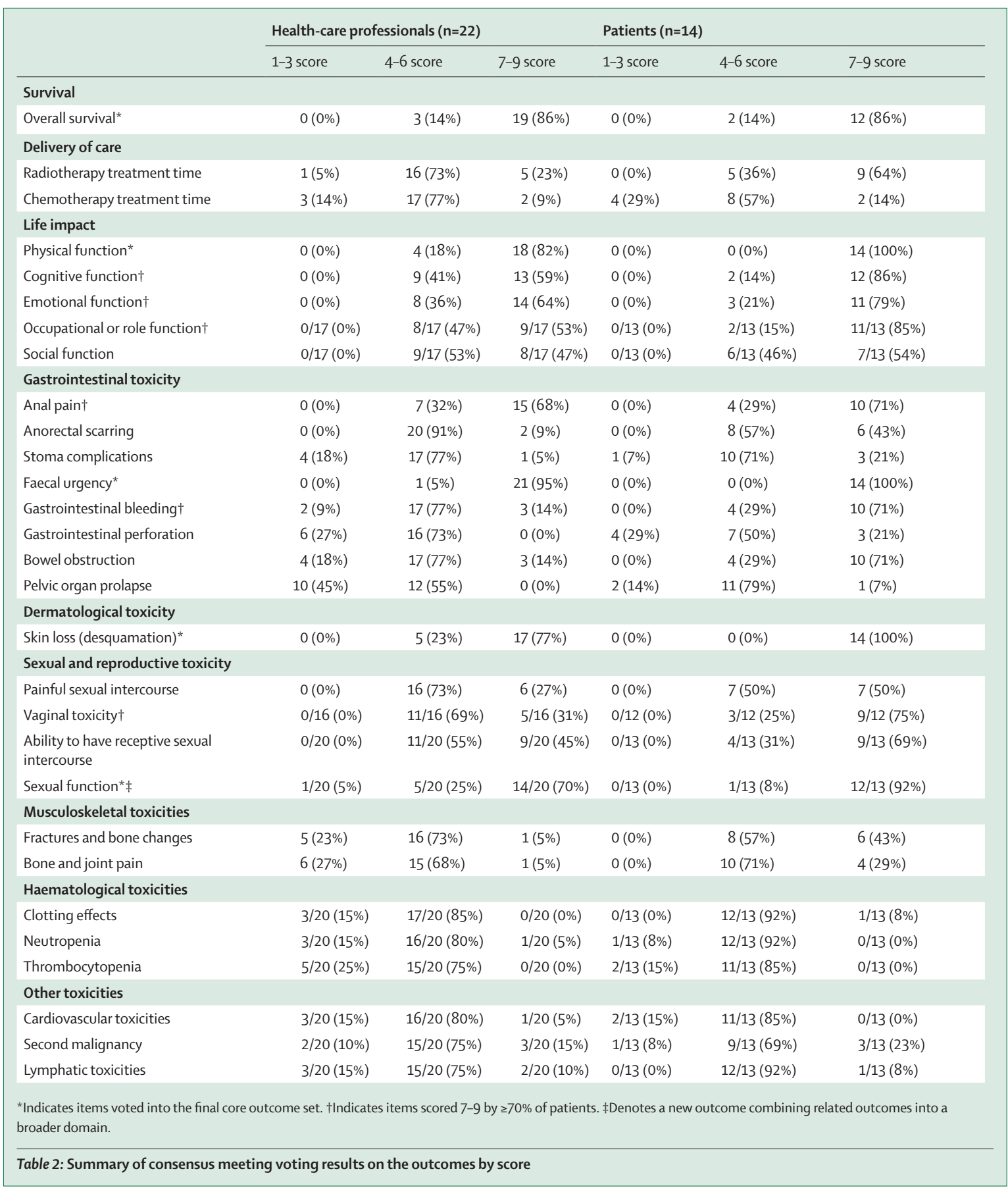

The remaining 14 outcomes in the final core outcome set were those that satisfied the criteria for inclusion in the final round of the Delphi survey.

During the analysis of the consensus meeting results, we noted one health-care professional had been erroneously self-allocated to the patient group. The effect of correcting this error was modelled. If that health-care professional had selected the correct stakeholder group, it is possible that one outcome (sexual function) might not have reached 7-9 scores by more than $70 \%$ of health-care professionals, and, therefore, it would not have been included in the core outcome set. Since only one of the 14 participants in the self-allocated patient group scored this outcome less than seven, the chance of correct self-allocation changing the results is only $7 \%$ (one of 14). No other outcomes could have been affected by this self-allocation error. However, the final core outcome set was reviewed and 
Panel: Final core outcome set

Disease activity

- Treatment response

- Local failure

- Regional failure

- Distant failure

- Disease progression

- Salvage surgery

Survival

- Overall survival

- Cancer-specific survival

- Disease-free survival

- Metastasis-free survival

- Progression-free survival

Toxicity

- Anal incontinence

- Faecal urgency

- Pelvic fistula

- Colostomy or ileostomy

- Skin loss

Life impact

- Physical function

- Sexual function

- Health-related quality of life

agreed by all participants at the close of the consensus meeting. Therefore, we recommend that the original results stand.

The final core outcome set (panel) includes 19 outcomes across four domains (six disease activity; five survival; five toxicity; three life impact). These were: treatment response, local failure, regional failure, distant failure, disease progression, and salvage surgery for disease activity; overall, cancer-specific, disease-free, metastasisfree, and progression-free survival; anal incontinence, faecal urgency, pelvic fistula, stoma, and skin loss for toxicity; and physical function, sexual function, and HRQoL for life impact.

The feedback questionnaire was completed by 17 (74\%) of 23 health-care professionals and all patients. All patients and 16 (94\%) of 17 health-care professionals were comfortable communicating their views during the meeting (one health-care professional was ambivalent). All patients and $15(88 \%)$ of 17 health-care professionals agreed that the meeting produced a fair result. Two health-care professionals deferred judgment until the final report was produced. Participants from both stakeholder groups commended the meeting for facilitating discussion between health-care professionals and patients.

\section{Discussion}

Our study provides the first international combined health-care professional and patient consensus on outcomes for trials in squamous cell carcinoma of the anus. All the included outcomes were identified as critically important by more than $70 \%$ of both patients and health-care professionals, using consensus methods to ensure equal representation of these groups. We recommend that all future trials evaluating chemoradiotherapy for squamous cell carcinoma of the anus use the CORMAC core outcome set as a framework for outcome selection.

We have not identified any other published core outcome sets for anal cancer. Glynne-Jones and colleagues $^{22}$ previously identified the need for consensus on outcome definitions in anal cancer trials, but made recommendations on the basis of the authors' views, without direct involvement of patients or the wider community of health-care professionals. By contrast, both patients and a broad range of health-care professionals have been involved in each stage of the development of this core outcome set.

In our initial systematic review, ${ }^{14}$ we identified more than ten different survival or survival composite outcome terms, all with varying definitions. Except overall survival, no survival outcome was reported in every randomised trial, and none has a single agreed definition. This heterogeneity reflects the lack of consensus (until now) on which survival endpoints to use other than overall survival.

There were some unexpected inclusions and exclusions in the final core outcome set. Colostomy-free survival, which has been commonly used in trials in this field, was not selected as a core outcome, but colostomy was. This illustrates a pitfall of creating composite outcomes; even when the events used to create a composite outcome are of interest, relevance of the composite cannot be assumed. The difficulties of defining progression-free survival and its validity as a marker for improved survival or QoL have been widely discussed, ${ }^{-11,14,23-25}$ however, progression-free survival is included in the CORMAC core outcome set, indicating that it holds relevance to both patients and health-care professionals. In the next phase of the project, we will work to agree standardised definitions for the included outcomes.

The new European Organisation for Research and Treatment of Cancer (EORTC) QoL module, ANL-27, ${ }^{26}$ is an anal-cancer-specific patient-reported outcome measure developed and validated in a large international cohort of patients, which identifies the patient-reported toxicity and functional outcomes that affect HRQoL in squamous cell carcinoma of the anus. The EORTC project aimed solely to evaluate the factors influencing HRQoL and did not evaluate patient (or health-care professional) views on survival or disease activity outcomes. By contrast, the present core outcome set will serve as an overall framework to capture a wide range of agreed outcomes in future trials. The outcomes in the CORMAC core outcome set were derived through a transparent, inclusive consensus process, from a 
comprehensive list of all possible outcomes across four domains (figure, panel), generated through systematic review and interviews with patients.

The CORMAC study has several strengths. Our method is coherent with recommendations from an international consensus $^{27}$ and was clearly defined in a protocol a priori. $^{16}$ Inclusion of both patients and health-care professionals at every stage ensured that outcomes in the final core set fairly represent their shared priorities. A unique strength of our consensus meeting, highlighted in the participant feedback, was directly bringing together patients and health-care professionals, enabling each group to hear the others' views and facilitating open discussion. We ensured that the views of both stakeholder groups were represented equally, despite differences in the number of participants in each group, by applying the same consensus criteria to electronic voting as was used in the Delphi survey. Our comprehensive and rigorous long-listing process ensured that outcomes across all domains (survival, disease activity, life impact including HRQoL, toxicity, and delivery of care) were considered during the consensus process.

There are some limitations to this study. Our project was done only in English, due to time and budgetary constraints, although our Delphi process included patient and health-care professional participants from 11 countries. The attrition rate for patient participants in the second round of our Delphi is slightly higher than that in other recent core outcome set projects, ${ }^{28-30}$ possibly affected by the recruitment methods used. To maximise international reach, we disseminated Delphi invitations via social media and group emails through patient advocacy groups, with $62 \%$ of all patient participants being recruited via these channels. This group had a higher attrition rate compared with those recruited via hospital sites (31\% vs 15\%), suggesting that participants recruited online were not as invested in the process as those recruited through personal contact.

It is important to acknowledge the interplay between outcomes in the toxicity and life impact domains. The toxicity domain relates to physiological outcomes (including symptoms), whereas the life impact domain relates to the functional items and composite measures of HRQoL. At the consensus meeting, both health-care professionals and patients described the functional impact of the included toxicity outcomes. However, patients also described the value of specific toxicity outcome data-eg, the incidence and duration of symptoms - in addition to measures of impact. Therefore, we feel that it is important to maintain the distinction between these two domains.

The life impact outcomes in the CORMAC core outcome set include physical and sexual function, as well as the composite measure of HRQoL. It is likely that the life impact and toxicity outcomes included in the CORMAC core outcome set are factors of HRQoL in squamous cell carcinoma of the anus, but identifying the determinants of HRQoL was not the aim of this project, and there are outcomes not included in the core outcome set that influence HRQoL, as described in the ANL-27 study. ${ }^{26}$ The concordance between the toxicity and life impact outcomes included in the CORMAC core outcome set and the question items included in ANL-27 makes it probable that ANL-27 will be recommended as the preferred measurement tool for these core outcomes. However, definitive recommendations cannot be made until full evaluation of the available tools has been completed in the next phase of the CORMAC project.

There were six outcomes that were not included in the final core outcome set (table 2) that were rated as critically important by patients, but not critically important by health-care professionals, at the consensus meeting. However, a core outcome set is a minimum set of outcomes that should be included in trials, in a particular field. The issues identified as of key importance to patients, including those not reaching the inclusion threshold in the core outcome set, can be used to aid the selection of additional outcomes of interest and to guide the research agenda going forward. A core outcome set should also be reviewed periodically to determine whether any excluded outcomes should be added or any included outcomes removed. ${ }^{12}$

Historically, toxicity outcome reporting in trials for squamous cell carcinoma of the anus (and in oncology in general) has been poor, with toxicity outcomes frequently reported only in non-specific terms such as gastrointestinal toxicity or acute toxicity. ${ }^{14}$ The clinician-reported Common Terminology Criteria for Adverse Events (CTCAE) system is the most widely used tool for measuring toxicity in oncology trials, ${ }^{31}$ including anal cancer trials. ${ }^{14}$ However, there are no guidelines for the clinical application of a given toxicity grading system, such as methods of patient screening or data collection, ${ }^{32}$ and trial reports rarely describe these methods in any detail..2 Clinician reporting of symptomatic toxicity outcomes has been shown to lack reliability ${ }^{33}$ and underestimate the incidence and severity of symptoms compared with patients' direct reports. ${ }^{34-36}$ Recognition of these issues has led to the development of new instruments for direct patient reporting of toxicity outcomes, such as PRO-CTCAE ${ }^{37}$ and eRAPID. ${ }^{38}$ However, such patient-reported outcome measures do not necessarily include outcomes that are important to patients, and the issue of selecting which toxicity outcomes to measure in a given trial has yet to be adequately addressed. The eRAPID system has selected several outcomes frequently experienced during treatment for five of the most common cancers. Therefore, toxicities encountered during treatment for rare cancers, such as anal cancer, might not be represented. The PRO-CTCAE system is derived from the CTCAE and includes a comprehensive library of 124 symptomatic toxicity outcomes from which trialists can construct bespoke patient-reported outcome measures by selecting 


\section{Search strategy and selection criteria}

Full details of the systematic review, including search strategy, databases, and selection criteria have been published elsewhere. ${ }^{14}$ Briefly, the systematic review identified 1243 outcomes from 101 studies trials and observational studies of chemoradiotherapy for squamous cell carcinoma of the anus. Outcomes and accompanying definitions were extracted verbatim from included studies and categorised into domains.

applicable question items. To date, there are no recommended outcome subsets specific to squamous cell carcinoma of the anus. The CORMAC core outcome set will facilitate selection of health-area-specific evaluation tools in future trials, by identifying the toxicities of critical importance to patients and health-care professionals, therefore increasing relevance and reducing redundancy.

Efforts will be needed to promote and monitor uptake of this core outcome set. The COMET initiative works to promote the use of core outcome sets, ${ }^{39}$ and trial funding bodies, regulatory authorities, and guideline development groups, such as the UK National Institute for Health Research, the European Medicines Agency, and the UK National Institute for Health and Care Excellence, now actively endorse the use of core outcome sets. Searches of trial registries identified five phase 2 and two phase 3 clinical trials of interventions for squamous cell carcinoma of the anus that are recruiting or opening soon. We recommend that the trial management groups of these studies review the CORMAC core outcome set to consider if any changes to trial outcome measurements should be made to accommodate the recommended core outcomes. The most recent of the phase 3 trials, PLATO, ${ }^{18}$ commenced recruitment in the UK in 2017, and aims to evaluate both dose de-escalation in early stages and dose escalation in locally advanced disease. There is already considerable overlap between the outcomes specified in the PLATO trial protocol and the CORMAC core outcome set.

Development of this core outcome set involved participation of stakeholders from 11 different countries; however, further work should be undertaken to validate this core outcome set more widely, especially in nonEnglish speaking populations. Finally, the CORMAC core outcome set describes which outcomes should be included in future clinical trials in squamous cell carcinoma of the anus. To ensure quality and consistency in measurement and reporting of these outcomes, in the next phase of this project we will work to agree standardised definitions and recommended measurement instruments for each outcome in the core outcome set, following the approach recommended by the Consensus-based Standards for the Selection of Health Measurement Instruments and COMET collaboration. ${ }^{40}$
The outcomes included in the CORMAC core outcome set represent the consensus opinion of an international group of patients, health-care professionals, and trialists, and addresses an unmet need: assisting trialists in the design, conduct, and reporting of trials. Ultimately, implementation of the CORMAC core outcome set will enhance the relevance of trial findings to health-care professionals, trialists, and patients.

\section{Contributors}

AGR and PRW conceived the project and are the joint principal investigators for the study. RF is the clinical research fellow and is responsible for management of the project. RF did the systematic review and Delphi process, and convened the consensus meeting. AGR, PRW, and CS provided supervision and have had input to all aspects of the project. AGR and PRW provided guidance on the systematic review, PRW provided guidance on the Delphi process and consensus meeting, and CS provided guidance on the patient interviews and qualitative analysis. RA, JB, JDN, RK, MPS, and DS-M formed the CORMAC study advisory group and along with AGR, CS, and PRW contributed to the preparation of material for population of the Delphi questionnaire. DS-M was a lead member of the study advisory group and supervised the formulation of clinical descriptions of disease activity and survival outcomes used in the Delphi process. The study advisory group and AGR participated in the consensus meeting. STB chaired and contributed to the planning of the consensus meeting with RF and PRW. CS and PRW assisted with facilitation of the consensus meeting. RF wrote the first draft of the manuscript and STB, CS, DS-M, AGR, and PRW have critically revised the manuscript. All authors have read, and confirm that they meet the International Committee of Medical Journal Editors (ICMJE) criteria for authorship.

\section{Declaration of interests}

PRW is a member of the COMET management group. All other authors declare they have no competing interests.

\section{CORMAC Study Advisory Group}

A G Renehan (Professor of Cancer Studies and Colorectal Surgeon, University of Manchester, Manchester), C Sanders (Reader in Medical Sociology, University of Manchester, Manchester), P R Williamson (Professor of Biostatistics and co-founder of COMET initiative, University of Liverpool, Liverpool), R Kochhar (radiologist, Cardiff University, Cardiff), J Brewer (specialist nurse, Christie National Health Service Foundation Trust, Manchester), J DeNardo (patient representative, Christie National Health Service Foundation Trust, Manchester), D Sebag-Montefiore (Professor of Clinical Oncology and Health Research, St James University Hospital, Leeds), R Adams (Professor of Clinical Oncology and Trials Research, Cardiff University and Velindre Cancer Centre, Cardiff), M P Saunders (Clinical Oncologist, Christie National Health Service Foundation Trust, Manchester).

\section{Consensus meeting participants}

Ane Appelt, Lynne Atwood, Graham Branagan, Nancy Baxter, Lindy Berkman, Angela Betsworth, Julie Brewer, Sara Brookes (chair), Rachel Cooper, Peter Coyne, David Crowe, Tamzin Cuming, Ian Curtis, Debbie Daley, Lucy Davidson, Jill DeNardo, Luc Dewit, Martyn Evans, Nicola Fearnhead, Ian Geh, Catherine Grierson, Vicky Goh, Marianne Guren, Rachel Hargest, Elaine Hughes, Helene Hutchings, Maggie Llewellyn, Sarah March, Victoria McFarlane, Dean Nixon, Andrew Renehan, Mark Saunders, Rick Saunders,

David Sebag-Montefiore, Carol Sleith, Neil Smart, Julia Young.

\section{Acknowledgments}

This paper presents independent research funded by the National Institute for Health Research under its Research for Patient Benefit Programme (grant reference number PB-PG-1013-32064). The views expressed are those of the authors and not necessarily those of the NHS, the NIHR or the Department of Health. This work was carried out as part of a PhD, funded in part by the Medical Research Council North West Hub for Trials Methodology Research (grant reference number MR/K025635/1). We acknowledge funding from the Christie 
charitable Pseudomyxoma Peritonei research fund, and the kind donations to this fund from patients, families, and friends. We thank Hannah Lennon for support with R script for Delphi analysis, Richard Crew for support with DelphiManager, and Heather Bagley for guidance on patient involvement at the consensus meeting. Thanks to Ruth Norris, Nasra Alam, and Shira Baram for helping with the consensus meeting. We thank all the patients and health professionals who have participated in the project.

\section{References}

1 Islami F, Ferlay J, Lortet-Tieulent J, Bray F, Jemal A. International trends in anal cancer incidence rates. Int J Epidemiol 2017; 46: 924-38

2 Nigro ND, Vaitkevicius VK, Considine BJ. Combined therapy for cancer of the anal canal: a preliminary report. 1974. Dis Colon Rectum 1993; 36: 709-11.

3 Nigro ND, Vaitkevicius VK, Buroker T, Bradley GT, Considine B. Combined therapy for cancer of the anal canal. Dis Colon Rectum 1981; 24: 73-75.

4 UKCCCR Anal Cancer Trial Working Party. Epidermoid anal cancer results from the UKCCCR randomised trial of radiotherapy alone versus radiotherapy, 5-fluorouracil, and mitomycin. Lancet 1996; 348: 1049-54.

5 Bartelink H, Roelofsen F, Eschwege F, et al. Concomitant radiotherapy and chemotherapy is superior to radiotherapy alone in the treatment of locally advanced anal cancer: results of a phase III randomized trial of the European Organization for Research and Treatment of Cancer Radiotherapy and Gastrointestinal Cooperative Groups. J Clin Oncol 1997; 15: 2040-49.

6 Ajani JA, Winter KA, Gunderson LL, et al. Fluorouracil, mitomycin, and radiotherapy vs fluorouracil, cisplatin, and radiotherapy for carcinoma of the anal canal: a randomized controlled trial. JAMA 2008; 299: 1914-21.

7 Peiffert D, Tournier-Rangeard L, Gérard J-P, et al. Induction chemotherapy and dose intensification of the radiation boost in locally advanced anal canal carcinoma: final analysis of the randomized UNICANCER ACCORD 03 trial. J Clin Oncol 2012; 30: 1941-48.

8 James RD, Glynne-Jones R, Meadows HM, et al. Mitomycin or cisplatin chemoradiation with or without maintenance chemotherapy for treatment of squamous-cell carcinoma of the anus (ACT II): a randomised, phase 3, open-label, $2 \times 2$ factorial trial. Lancet Oncol 2013; 14: 516-24.

9 Flam M, John M, Pajak TF, et al. Role of mitomycin in combination with fluorouracil and radiotherapy, and of salvage chemoradiation in the definitive nonsurgical treatment of epidermoid carcinoma of the anal canal: results of a phase III randomized intergroup study. J Clin Oncol 1996; 14: 2527-39.

10 Sekhar H, Zwahlen M, Trelle S, et al. Nodal stage migration and prognosis in anal cancer: a systematic review, meta-regression, and simulation study. Lancet Oncol 2017; 38: 1348-59.

11 Glynne-Jones R, Adams R, Lopes A, Meadows H. Clinical endpoints in trials of chemoradiation for patients with anal cancer Lancet Oncol 2017; 18: e218-27.

12 Williamson PR, Altman DG, Bagley H, et al. The COMET Handbook: version 1.0. Trials 2017; 18 (suppl 3): 280.

13 Williamson PR, Altman DG, Blazeby JM, et al. Developing core outcome sets for clinical trials: issues to consider. Trials 2012; 132: 1-8.

14 Fish R, Sanders C, Ryan N, Van der Veer S, Renehan AG, Williamson PR. Systematic review of outcome measures following chemo-radiotherapy for the treatment of anal cancer (CORMAC). Colorectal Dis 2018; 20: 371-82.

15 Kichenadasse G, Zakaria J, Rodda DJ, et al. Squamous cell carcinoma of the anal canal at The Queen Elizabeth Hospital: a local experience. Asia Pac J Clin Oncol 2007; 3: 214-18.

16 Fish R, Sanders C, Williamson PR, Renehan AG. Core outcome research measures in anal cancer (CORMAC): protocol for systematic review, qualitative interviews and Delphi survey to develop a core outcome set in anal cancer. BMJ Open 2017; 7: e018726

17 COMET DelphiManager. 2018. http://www.comet-initiative.org/ delphimanager/index.html. (accessed June 13, 2018).
18 PersonaLising Anal cancer radioTherapy dOse- Incorporating ACT3, ACT4 and ACT5. 2016. http://medhealth.leeds.ac.uk/ info/430/solid_tumours/2210/plato (accessed Dec 30, 2016).

19 Kirkham JJ, Gorst S, Altman DG, et al. Core Outcome Set-STAndards for Reporting: The COS-STAR Statement. PLoS Med 2016; 13: e1002148.

20 Core Outcome Research Measures in Anal Cancer (CORMAC). 2015. http://www.comet-initiative.org/studies/details/7812018. (accessed June 13, 2018)

21 Dodd S, Clarke M, Becker L, Mavergames C, Fish R, Williamson PR. A taxonomy has been developed for outcomes in medical research to help improve knowledge discovery. J Clin Epidemiol 2017; 96: 84-92.

22 Glynne-Jones R, Adams RA, Jitlal M, Meadows H. End points in anal cancer: hopes for a common language. J Clin Oncol 2014 32: $1281-82$.

23 Oxnard GR, Morris MJ, Hodi FS, et al. When progressive disease does not mean treatment failure: reconsidering the criteria for progression. J Natl Cancer Inst 2012; 104: 1534-41.

24 Booth CM, Eisenhauer E. Progression-free survival: meaningful or simply measurable? J Clin Oncol 2012; 30: 1030-33.

25 Shi Q, Sargent D. Meta-analysis for the evaluation of surrogate endpoints in cancer clinical trials. Int J Clin Oncol 2009; 14: 102-11.

26 Sodergren SC, Johnson CD, Gilbert A, et al. Phase I-III development of the EORTC QLQ-ANL27, a health-related quality of life questionnaire for anal cancer. Radiother Oncol 2018; 126: 222-28.

27 Kirkham JJ, Gorst SL, Altman DG, et al. Core Outcome Set-STAndards for Development: the COS-STAD recommendations. PLoS Med 2017; 14: e1002447.

28 MacLennan S. A core outcome set for localised prostate cancer effectiveness trials: protocol for a systematic review of the literature and stakeholder involvement through interviews and a Delphi survey. Trials 2015; 16: 76.

29 McNair AG, Whistance RN, Forsythe RO, et al. Core outcomes for colorectal cancer surgery: a consensus study. PLoS Med 2016; 13: e1002071.

30 Avery KNL, Chalmers KA, Brookes ST, et al. Development of a core outcome set for clinical effectiveness trials in esophageal cancer resection surgery. Ann Surg 2017; 267: 700-10.

31 Basch E, Reeve BB, Mitchell SA, et al. Development of the National Cancer Institute's patient-reported outcomes version of the common terminology criteria for adverse events (PRO-CTCAE). J Natl Cancer Inst 2014; 106: dju244.

32 Trotti A, Bentzen SM. The Need for Adverse Effects Reporting Standards in Oncology Clinical Trials. J Clin Oncol 2004; 22: 19-22.

33 Atkinson TM, Li Y, Coffey CW, et al. Reliability of adverse symptom event reporting by clinicians. Qual Life Res 2012; 21: 1159-64.

34 Pakhomov SV, Jacobsen SJ, Chute CG, Roger VL. Agreement between patient-reported symptoms and their documentation in the medical record. Am J Manag Care 2008; 14: $530-39$

35 Fromme EK, Eilers KM, Mori M, Hsieh YC, Beer TM. How accurate is clinician reporting of chemotherapy adverse effects? A comparison with patient-reported symptoms from the Quality-of-Life Questionnaire C30. J Clin Oncol 2004; 22: 3485-90.

36 Basch E. The missing voice of patients in drug-safety reporting. N Engl J Med 2010; 362: 865-69.

37 Dueck AC, Mendoza TR, Mitchell SA, et al. Validity and Reliability of the US National Cancer Institute's Patient-Reported Outcomes Version of the Common Terminology Criteria for Adverse Events (PRO-CTCAE). JAMA Oncol 2015; 1: 1051-59.

38 Holch P, Warrington L, Bamforth LCA, et al. Development of an integrated electronic platform for patient self-report and management of adverse events during cancer treatment. Ann Oncol 2017; 28: 2305-11.

39 COMET. COS Uptake and Endorsement. 2018. http://www.cometinitiative.org/cosuptake2018. (accessed June 13, 2018)

40 Prinsen CA, Vohra S, Rose MR, et al. How to select outcome measurement instruments for outcomes included in a "Core Outcome Set" — a practical guideline. Trials 2016; 17: 449.

(C) 2018 Elsevier Ltd. All rights reserved. 